\title{
Diseño de un proyecto de traducción para desarrollar la competencia temática
}

\author{
Design of a Translation Project to Develop the Thematic Translation Competence \\ Elaboração de um projeto de tradução para desenvolver a competencia temática
}

Angela Patricia Morales Santibáñez * (https://orcid.org/0000-0002-5463-0206)

Departamento de Lingüística y Literatura, Facultad de Humanidades, Universidad

de Santiago de Chile, Santiago, Chile

Recibido: $27-09-17$

Revisado: $30-10-17$

Aceptado: $21-11-17$

Publicado: $18-12-17$
RESUMEN. En el presente artículo se describe la etapa inicial de un Proyecto de Innovación Docente de la Universidad de Santiago de Chile, fase que incluye el diseño del proyecto y los alcances metodológicos para su implementación y evaluación. Expertos de distintas unidades académicas asignaron a los estudiantes encargos de traducción reales y los asesorarán para aportar al desarrollo de la competencia temática. El proyecto será implementado en la asignatura de Traducción especializada en Ciencias Sociales inglés-español ( $4^{\circ}$ año) de la carrera de Licenciatura en Lingüística Aplicada a la Traducción durante el segundo semestre de 2017. La propuesta se basa en los postulados de Kiraly (2000), quien afirma que la manera más efectiva de que un estudiante adquiera competencias profesionales es entregarle oportunidades para que interactúe con sus compañeros y con expertos. En el contexto del proyecto se diseñaron diversas pautas metodológicas e instrumentos de evaluación con diferentes intencionalidades, temporalidades y agentes, para responder a la necesidad de obtener una apreciación global del proceso en su conjunto. La aplicación de esta estrategia didáctica permitirá sistematizar una práctica que, si bien se ha utilizado previamente en la formación de traductores en la Universidad de Santiago, no cuenta con un diseño formal e instrumentos de evaluación concretos que permitan replicarla en otras asignaturas de traducción especializadas a nivel local y nacional.
Palabras clave:

formación de traductores, proyecto de traducción, competencia temática, asesoría de expertos, evaluación 
ABSTRACT. This article describes the first step in a Teaching Innovation Project in Universidad de Santiago de Chile, which includes the project's design and the methodological aspects required for its implementation and evaluation. Experts from different academic units will give students real translation assignments (specified in a translation brief) and will support them in the process so as to contribute to the development of their thematic translation competence. This project will be implemented in the Specialized Social Science English-Spanish Translation course (4th year) of the Bachelor of Linguistics Applied to Translation degree program during the September 2017 semester. The proposal is based on contributions from Kiraly (2000) stating that the most effective way for students to develop professional competences is by offering them opportunities to interact with their classmates and with experts. Within this project, various methodological guidelines and assessment instruments-with different purposes, applied at different times, and from distinctive agents-were designed so as to meet the need to obtain a global picture of the entire process. The implementation of this didactic strategy will contribute to the systematization of a teaching practice which, although being used in translators' training in Universidad de Santiago, lacks a formal design and specific assessment instruments that would allow replicating it in other specialized translation courses at a local and national level.

\footnotetext{
Keywords:

translators'

training,

translation

project,

thematic

translation

competence, experts'

consultancy,

evaluation
}

RESUMO. Neste estudo, descreve-se o estágio inicial de um Projeto de Inovação Docente da Universidade de Santiago do Chile, fase que inclui a elaboração do projeto e os alcances metodológicos para sua execução e avaliação. Especialistas de diferentes unidades acadêmicas encomendaram aos estudantes trabalhos de tradução reais e os assessoraram para contribuir para o desenvolvimento da competência temática. 0 projeto será a plicado no curso de Tradução Especializada em Ciências Sociais inglês-espanhol ( $4^{\circ}$ ano) do curso de Licenciatura em Linguística Aplicada à Tradução durante o segundo semestre de 2017. A proposta baseia-se nos postulados de Kiraly (2000), quem propõe que a forma mais efetiva para que o estudante adquira competências professionais é brindar-lhe oportunidades para que possa interagir com seus colegas e com especialistas. No âmbito do projeto, elaboraram-se diversas pautas metodológicas e instrumentos de avaliação com diferentes propósitos, temporalidades e agentes, para responder à necessidade de obter-se uma apreciação global do processo no todo. A utilização desta estratégia didática permitirá sistematizar uma prática que, mesmo tendo sido utilizada previamente na formação de tradutores na Universidade de Santiago, não conta com um desenho formal e instrumentos de avaliação concretos que permitam replicá-la em outras matérias de tradução especializada, ora local, ora nacional.
Palavras-chave: formação de tradutores, projeto de tradução, competência temática, assessoria de especialistas, avaliação 
En Chile se ha percibido la necesidad de un cambio de enfoque en la metodología tradicional de enseñanza de la traducción (Hurtado, 2007; Marco, 2004). Algunas universidades que cuentan con el programa de traducción han diseñado estrategias de aprendizaje activo basadas en los principios del aprendizaje por problemas y de la gestión de proyectos (Lazo \& Zachary, 2008). Sin embargo, no hay otras evidencias de propuestas formales de estrategias de aprendizaje activo que los profesores puedan utilizar en cursos de traducción especializada. En la carrera de Licenciatura en Lingüística Aplicada a la Traducción Inglés-Portugués/Japonés de la Universidad de Santiago la formación se ha mantenido centrada en el profesor como encargado de seleccionar textos, de resolver dudas durante el proceso y de evaluar el producto final. Generalmente, los estudiantes no cuentan con la asesoría de expertos en las áreas disciplinares donde se generan los textos como recurso para producir una versión traducida efectiva. Este proyecto surge de la necesidad de introducir estrategias de aprendizaje activo para la formación de traductores (Kiraly, 2000) y consiste en la traducción colaborativa al español de diferentes textos escritos originalmente en inglés, de áreas relacionadas con las ciencias sociales, que tienen una aplicación práctica en procesos de investigación y de enseñanza-aprendizaje dentro de la universidad. Los textos fueron seleccionados por expertos quienes, además, asesorarán a los estudiantes a través de interacciones presenciales y virtuales. El análisis permitirá identificar cómo la interacción con los expertos aporta al desarrollo de la competencia temática (Kelly, 2002) de los estudiantes, con el objetivo de producir una traducción efectiva según el encargo (Nord, 1991). El proyecto será implementado en la asignatura de traducción especializada en ciencias sociales ( $4^{\circ}$ año), el segundo semestre del año 2017 y contempla el diseño y la aplicación de diferentes instrumentos de evaluación, con énfasis en el desarrollo de la competencia temática. Se espera que este proyecto se constituya en una herramienta didáctica que pueda ser utilizada en diferentes asignaturas de traducción especializada, en la Universidad de Santiago y en otras universidades chilenas y que los instrumentos de evaluación generados sirvan como nuevos recursos para evaluar las versiones producidas por los alumnos dentro del contexto académico local y nacional. Actualmente el proyecto se encuentra en etapa de diseño de instrumentos de evaluación y próximo a ser implementado en la asignatura correspondiente.

\section{Fundamentos teóricos}

A partir de la década de 1990 surge en Europa, en el área de la traducción, la necesidad de buscar nuevas estrategias de aprendizaje activo en la formación de traductores que tengan impacto en el proceso traductor y no solo en el producto de dicho proceso (Kussmaul, 1995). A partir de entonces se han desarrollado varios estudios orientados a promover estrategias constructivistas para la formación de traductores. Paralelamente, la reflexión llevada a cabo con motivo de la implantación del Espacio Europeo de Enseñanza Superior ha puesto de manifiesto la importancia de un cambio de enfoque en la metodología tradicional (Hurtado, 2007; Marco, 2004). En Chile la situación no es diferente y algunas universidades han implementado estrategias de aprendizaje activo como el aprendizaje por problemas y la gestión de proyectos. (Lazo \& Zachary, 2008). Sin embargo, la carrera de Licenciatura en Lingüística Aplicada a la Traducción Inglés-Portugués/Japonés de la Universidad de Santiago no cuenta con una propuesta formal de estrategias de aprendizaje activo que los profesores puedan utilizar en los cursos de traducción especializada que dictan y es ese hecho el que motiva la presentación de este proyecto de innovación. 
La propuesta se basa en los postulados de Kiraly (2000), quien plantea que es necesario cambiar el foco de atención en las clases de traducción de la transmisión unidireccional de conocimientos del profesor al alumno a una interacción multidireccional entre todos quienes participan en el proceso. Según este autor, desde la perspectiva del socioconstructivismo, la manera más efectiva de lograr que un estudiante adquiera competencias profesionales es entregarle oportunidades para que interactúe con sus compañeros y con expertos y así construya su aprendizaje de manera colectiva e individual. El ambiente constructor del aprendizaje se caracteriza por una auténtica actividad reflexiva, distribución del conocimiento y de autoridad y niveles de autonomía que aumentan gradualmente en el estudiante. Por otra parte, según Kelly (2002) el trabajo colaborativo es clave para activar prácticamente todas las competencias que guían la formación del traductor: la competencia comunicativa y textual, la competencia cultural e intercultural, la competencia temática, la competencia profesional e instrumental, la competencia psicofisiológica y actitudinal, la competencia interpersonal y la competencia estratégica. La adquisición de estas competencias de manera compartida con otros sujetos permitirá que el futuro traductor realice de forma efectiva su labor traductora y lo ayudará a perfilar su identidad profesional (Muñoz, 2015). El proyecto colaborativo propuesto pretende aportar al desarrollo de la competencia temática a partir de la asesoría obtenida del experto. Esta competencia es fundamental en la traducción de textos especializados y comprende los conocimientos básicos sobre los campos temáticos en los que trabaja el traductor.

La propuesta sigue, además, los postulados funcionalistas de la traductología (House, 1997; Nord, 1991, 2009), los cuales sitúan la actividad traductora en las interacciones comunicativas que se producen en situaciones delimitadas con respecto a espacio y tiempo. Esto significa que cada situación comunicativa ocurre dentro de ciertas dimensiones históricas y culturales que condicionan el comportamiento verbal de los agentes, los conocimientos y expectativas que unos tienen de los otros, su valoración de la situación, el punto de vista que adoptan al mirarse mutuamente y al mundo. Desde esta perspectiva, la efectividad de la traducción está condicionada por el propósito comunicativo que cumple en un momento determinado. En otras palabras, la traducción será efectiva si logra cumplir las funciones comunicativas definidas en el "encargo de traducción”, que describe la situación para la que se destina el texto meta y las características específicas de ese acto comunicativo en particular.

\section{METODOLOGÍA CONSIDERADA PARA EL PROYECTO}

El proyecto pretende aportar al desarrollo de la competencia temática de los alumnos que trabajan colaborativamente en la traducción de un texto especializado del área de las Ciencias Sociales, de aproximadamente 3.000 palabras, asignado por un experto en el tema, quien los asesorará durante el proceso. El análisis estará orientado a relacionar la asesoría del experto con el desarrollo de la competencia temática y se articulará en torno a conceptos clave dentro de los textos. El proyecto se ejecutará en la asignatura de traducción especializada en Ciencias Sociales inglés-español, al inicio de este curso dictado el segundo semestre. Se seleccionó esta asignatura para la ejecución del proyecto debido a que es la primera en la malla curricular de la carrera relacionada con traducción especializada y a que en ella se trabajan textos de complejidad media, adecuada para una primera aplicación. Además, la profesora responsable del proyecto dicta esta asignatura en las dos secciones y en la fase inicial 
de diseño recibió propuestas de expertos voluntarios para traducir textos que provenían precisamente de áreas relacionadas con las Ciencias Sociales (Sociología, Psicología de la Educación y Relaciones Internacionales). Los estudiantes trabajarán en el proyecto durante un mes con una dedicación semanal de cuatro horas directas y tres de trabajo autónomo. Esta asignatura corresponde al $4^{\circ}$ año de la carrera de lingüística aplicada a la traducción inglés-portugués/japonés, que tiene una duración de cinco años, y constituye el primer acercamiento formal a la traducción especializada de los alumnos que ya cuentan con una base teórica y práctica relativamente sólida para traducir. Cada sección tiene un promedio de 15 alumnos, por lo que al dividir aleatoriamente cada curso en grupos de tres alumnos resultan 10 grupos de trabajo en total, cada uno asesorado por un experto. Se seguirá la lógica del diseño por encuesta longitudinal, con recogida de datos al inicio del proyecto, durante el proceso y al final de él, todos ellos dirigidos a analizar el desarrollo de la competencia temática en los estudiantes, mediado por el experto. Los datos iniciales se obtendrán a través de un cuestionario de diagnóstico para evaluar el manejo de los conceptos clave identificados. Durante el proceso, y al final de él, se recolectarán datos a través de los instrumentos de evaluación que se describen en el siguiente apartado:

\section{Diseño de pautas metodológicas e instrumentos de evaluación}

El guion del proyecto (ver Apéndice A). La primera pauta diseñada fue el guion del proyecto. Este instrumento se define como el elemento que explicita ordenadamente los contenidos que se trabajarán en el proyecto y explica a los alumnos todas las etapas que se recorrerán durante su ejecución, junto con los plazos establecidos. El guion diseñado fue sometido a una validación de juicio experto a cargo de profesores de la carrera que dictan cursos de traducción, quienes revisaron y entregaron sugerencias en cuanto a su contenido. Una vez validado el guion del proyecto, e implementadas las sugerencias de los expertos, se inició el diseño de los distintos instrumentos de evaluación.

Diseño de instrumentos de evaluación: Fundamentos teóricos. Los pilares teóricos que sirvieron como base para el diseño de los instrumentos de evaluación son los siguientes: el modelo revisado de evaluación de traducciones de House (1997) así como propuestas concretas de instrumentos de evaluación de traducciones de Marais (2013), Rojas (2004) y Medadian y Nezhadansari (2015), además de las aportaciones de la didáctica general (Ahumada, 2001).

El modelo de evaluación de House (1997) toma como base propuestas que nacen de diversos enfoques teóricos en traductología. En su modelo revisado, House (1997) concibe la traducción como una oferta informativa de un texto en lengua origen por un texto equivalente en lengua meta desde el punto de vista semántico, textual y pragmático. Desde esta perspectiva, el texto está ligado indisolublemente a la situación comunicativa donde se genera y esta condiciona su estructura textual y sus rasgos lingüísticos. En consecuencia, la calidad de la traducción de un texto en particular estaría relacionada con el grado en el cual ese texto es equivalente al original en cuanto reproduce los rasgos textuales y causa en los destinatarios el mismo efecto pragmático que el texto original.

El modelo considera los conceptos de campo, tenor y modo propuestos por Halliday (1985). La dimensión "campo" se relaciona con el tema y el contenido del texto, reflejados en diferentes grados de especificidad en las elecciones léxicas. Como segundo elemento, el "tenor" caracteriza a los participantes 
en el acto comunicativo y su relación en términos de poder y distancia social, además de su ubicación temporal y geográfica. También abarca la actitud social de los participantes y los diferentes registros donde se mueven en el acto comunicativo (formal, informal etc.). Finalmente, el modo se refiere al canal de comunicación (simple o complejo) y al grado de interacción entre el emisor y el receptor.

House (1997) distingue entre traducción patente (overt translation) y traducción encubierta (covert translation). Según Waddington (1999), la traducción patente está íntimamente ligada a la lengua y cultura original. Este concepto nace del movimiento "hacia el autor" en traducción identificado por Schleiermacher (2000) y puede asociarse, también, con la traducción extranjerizante propuesta por Venuti (1995) y con la traducción documental que identifica Nord (1991). Este tipo de traducción se utilizaría para textos que no pierden su valor con el paso del tiempo (por ejemplo, obras literarias, discursos políticos, etc.). Por otra parte, la traducción encubierta se define como aquella que funciona como un texto original en la cultura de llegada y podemos asociarla con el movimiento "hacia el lector" identificado por Schleiermacher (2000), con la traducción domesticante extranjerizante propuesta por Venuti (1995) y con la traducción instrumental caracterizada por Nord (1991). Algunos ejemplos de traducciones encubiertas serían manuales de maquinarias, instrumentos jurídicos, etc.

Según House (1997), para evaluar una traducción es fundamental considerar la diferenciación entre traducción abierta y encubierta, ya que ambas requieren un análisis crítico diferente. Por ejemplo, en el caso de la traducción abierta, la transferencia es más directa y por ello impone menos complejidades para la evaluación. Por otra parte, para evaluar la traducción encubierta se debe poner atención en las presunciones culturales y en las preferencias comunicativas de los participantes.

Los textos trabajados en el proyecto podrían clasificarse como "traducción encubierta", en cuanto funcionarán como instrumentos de comunicación independientes en la cultura término y, por lo tanto, los instrumentos de evaluación diseñados estarán orientados a este tipo de traducción. Al iniciar el proceso de traducción se reflexionará con los estudiantes respecto del tema y contenido del texto, la caracterización de los participantes en el acto comunicativo y la relación social de los mismos.

Diseño de instrumentos de evaluación. Al concebir la traducción como un acto de comunicación, varios autores han dejado ver la necesidad de diseñar instrumentos de evaluación que entreguen una mirada al proceso y no solo al producto (House, 1997; Larose, 1989; Nord, 1991). Para satisfacer esta necesidad se ha propuesto el diseño de evaluaciones holísticas, que permitan una apreciación global sobre el proceso en su conjunto y que complementen los métodos analíticos tradicionales. La necesidad de diseñar diversos instrumentos de evaluación holísticos y analíticos para el presente proyecto de traducción surge al entender la evaluación como una etapa fundamental del proceso de enseñanzaaprendizaje, cuyo objetivo principal es entregar al estudiante información sobre este proceso de manera que pueda tomar decisiones oportunas para mejorarlo en caso necesario (Ahumada, 2001). Según Medadian y Nezhadansari (2015), esta apreciación global debe diversificarse en diferentes agentes evaluadores, con distintas funciones y en diversas etapas del proceso. A partir de este fundamento teórico se procedió a diseñar los instrumentos de evaluación con diferentes intencionalidades (formativa, sumativa), temporalidades (inicial, procesual, final) y agentes (heteroevaluación de profesor y de experto y coevaluación) que se describirán a continuación. 
Evaluación formativa inicial. La evaluación formativa inicial o evaluación diagnóstica, es aquella que se aplica al comienzo del proceso de enseñanza-aprendizaje para detectar la situación de partida y visibilizarla de manera que los estudiantes tomen conciencia de la base sobre la que se cimentarán los aprendizajes que se construirán de ahí en adelante.

En el contexto del proyecto, la evaluación diagnóstica permitirá detectar el nivel de familiaridad de los estudiantes con los conceptos fundamentales del texto para mostrar cuáles son aquellos más débiles que es necesario reforzar; así como recuperar cualquier tipo de conocimiento previo que cimente la construcción de nuevos aprendizajes.

La etapa de diseño de la evaluación diagnóstica comenzó con la lectura profunda de los textos asignados por los expertos (ver Apéndice B) y el análisis del encargo de traducción para identificar las características específicas de cada acto comunicativo en particular. Después de la lectura de los textos se identificaron los conceptos clave y, a partir de ellos, se crearon mapas conceptuales que guiaron la elaboración del instrumento. La lectura profunda del texto orientó la selección de cinco conceptos clave que se incorporaron en la evaluación. Este instrumento será entregado a los estudiantes, junto con el texto asignado al comienzo del proceso, y está constituido por dos secciones que se complementan entre sí. La primera es una selección de cinco conceptos clave para que los estudiantes los definan en sus propias palabras con el apoyo del texto completo. La segunda parte está constituida por cuatro preguntas de comprensión de lectura relacionadas con las ideas fundamentales del texto.

\section{Evaluaciones procesuales}

\section{1) Evaluación formativa}

Se diseñaron instrumentos con intencionalidad formativa para valorar el proceso, obtener información sobre el avance e identificar fortalezas y debilidades que reforzar. El diseño de estos instrumentos se concibió a partir de las aportaciones de Ahumada (2001), quien plantea que al evaluar permanentemente los aprendizajes del alumno se pueden detectar situaciones educativas que favorecen sus aprendizajes y disponer de los medios didácticos adecuados para superar inconvenientes en el momento oportuno para facilitar el logro del objetivo final. Un proyecto de traducción, como proceso, necesita evaluaciones formativas cualitativas que permitan visibilizar su desarrollo y hacer ajustes continuos para asegurar un buen resultado final. Los instrumentos diseñados son de tipo holístico; es decir, aquella evaluación global que, de alguna manera, nos permite valorar el desarrollo de las competencias del estudiante de traducción de manera continua durante su desarrollo (Toledo, 2010). A partir de los modelos propuestos por Rojas (2004) se diseñaron los siguientes instrumentos de evaluación formativa:

\section{Registros de fortalezas y debilidades del profesor y del experto (ver Apéndices C y D)}

Se trata de dos tablas separadas donde el profesor y el experto temático asignado registrarán sus comentarios respecto de las fortalezas y debilidades que perciben en el desarrollo del proyecto. Se registrarán, por una parte, las observaciones del profesor que se relacionarán con los dominios cubiertos por la evaluación sumativa final (comprensión, producción y transferencia) 
y los comentarios del experto relacionados específicamente con el desarrollo de la competencia temática (dominio del tema, adaptación al destinatario e interacción con el asesor). Además, ambos agentes evaluadores tendrán un espacio destinado a comentarios abiertos.

\section{2) Evaluación sumativa}

Bitácora de reflexión del estudiante (ver Apéndice E). Se define como una narración libre que registrará las reflexiones de los estudiantes en torno al proceso en tres momentos definidos: principio (primera semana de trabajo), mitad (final de segunda semana), término (final del proceso). El objetivo es tomar conciencia sobre el camino recorrido e internalizarlo, concentrándose en el desarrollo de la competencia temática. La bitácora se elaboró en torno a tres preguntas diferentes para cada una de las tres etapas del proceso: algunas de ellas de elaboración propia y otras adaptadas del cuestionario sobre el proceso traductor propuesto por Umaña y Suárez (2011) como uno de los instrumentos utilizados para medir la competencia traductora. Se plantearon preguntas como: ¿Cuáles son los principales problemas enfrentados en relación con el dominio del tema? ¿Qué recursos y estrategias fueron útiles para resolver los problemas relacionados con el dominio del tema? y ¿Qué aprendí para el futuro respecto de la competencia temática? La bitácora de reflexión se evaluará mediante una pauta y tendrá una ponderación de $20 \%$ en la evaluación sumativa final.

Evaluaciones sumativas finales. La finalidad de estas evaluaciones es valorar el producto final del proceso terminado. El proyecto colaborativo contará con tres evaluaciones sumativas finales: una a cargo del profesor del curso, otra a cargo del experto asesor y una coevaluación. Se diseñó una rúbrica de evaluación para el profesor y una escala de apreciación con comentarios del experto (con ponderaciones de $40 \%$ y $30 \%$, respectivamente). El 30\% restante estará reservado a la evaluación de la bitácora de reflexión (20\%) y a la coevaluación (10\%).

a) Rúbrica de evaluación del profesor (ver Apéndice F): Como instrumento de evaluación del profesor, se diseñó una rúbrica que valora el texto traducido en tres grandes dimensiones: comprensión del texto original, producción del texto meta y transferencia. Se identificaron estas dimensiones como principales indicadores de la calidad de un texto traducido siguiendo las propuestas de Rodríguez (2013). Esta autora se basa en los aportes de autores como Mossop (2001) y Parra (2005) para proponer ciertos parámetros que guían el proceso de revisión de textos traducidos: contenido, lenguaje, finalidad y presentación. Este referente teórico recomienda el enfoque didáctico por tareas y el constructivista para adquirir competencias relacionadas con la revisión de textos traducidos, con el objetivo de alinearse con los cambios metodológicos y el rol de los alumnos en los nuevos planes de estudios universitarios en España. Los criterios de evaluación se adaptaron al contexto de este proyecto colaborativo; pero son de naturaleza general y, por lo tanto podrían utilizarse también en otros proyectos de traducción especializada en un contexto docente.

b) Escala de apreciación del experto (ver Apéndice G): Como instrumento de evaluación del experto se diseñó una escala de apreciación con indicadores que pretenden evaluar directamente el avance en el desarrollo de la competencia temática evidenciado por los estudiantes, así como la adaptación al destinatario y componentes actitudinales específicos 
de la interacción, como responsabilidad, puntualidad y respeto. Los valores numéricos se complementan por comentarios abiertos del experto.

c) Coevaluación (ver Apéndice H): La coevaluación consiste en la evaluación mutua, conjunta, de una actividad o de un trabajo determinado realizado entre varios estudiantes. Tras un trabajo en equipo, cada uno valora lo que le ha parecido más interesante de los otros. Se pueden utilizar diversos instrumentos de coevaluación. Esta, junto con otras estrategias metodológicas de evaluación, es una interesante alternativa complementaria para evaluar determinadas competencias de los estudiantes universitarios, ya que permite la participación de los estudiantes y es esta participación la que según Boud y Falchikov (2006) puede ayudar a motivar, mejorar y consolidar el aprendizaje, además de favorecer aprendizajes que perduran a lo largo de la vida. En el caso específico del presente proyecto colaborativo, la coevaluación puede entregar información sobre el proceso en la que solo tienen acceso los estudiantes que participaron directamente en él y mostrarnos las direcciones por dónde debemos encaminar las mejoras, Este instrumento tendrá una ponderación de $10 \%$ en la evaluación sumativa final y se evaluará mediante una escala de apreciación complementada con comentarios, orientada a la actuación de la competencia temática.

\section{CONCLUSIÓN}

El diseño de este proyecto colaborativo nace de la necesidad identificada por varios autores (Hurtado, 2007; Kussmaul, 1995; Marco, 2004) de implementar en la formación de traductores estrategias de aprendizaje activo que permitan que el estudiante asuma un rol protagónico para desarrollar sus competencias y que tengan impacto en el proceso traductor. El proyecto está dirigido a desarrollar específicamente la competencia temática, percibida como una competencia clave en traducción especializada, que puede perfeccionarse mediante el trabajo colaborativo con el apoyo del iniciador del encargo como especialista en el tema, en una situación de trabajo real. Como tal, se constituye como un aporte a la formación de traductores en Chile, ya que su diseño y los instrumentos de evaluación elaborados podrán utilizarse en otras asignaturas de traducción especializada en diferentes áreas dentro de la Universidad de Santiago y en otras universidades chilenas.

La etapa de diseño de instrumentos de evaluación de diferentes tipos para este proyecto de traducción permitió volver a reflexionar sobre los distintos roles que cumple la evaluación en los procesos de aprendizaje. Frecuentemente nos enfrentamos con el rol tradicional de la evaluación para medir el aprendizaje; no obstante su objetivo fundamental es mostrar la evolución del proceso; de manera que los estudiantes puedan tomar medidas oportunas para superar los obstáculos enfrentados. En el caso específico de la traducción, varios autores han dejado ver la necesidad de diseñar instrumentos de evaluación que entreguen una mirada al proceso y no solo al producto (House, 1997; Larose, 1989; Nord, 1991). Los instrumentos de evaluación diseñados para el proyecto servirán como ayuda para que los estudiantes internalicen su propio proceso y lo regulen de manera que se produzcan las mejoras deseadas de manera oportuna. Es importante, también, que se considere la mirada de otros agentes involucrados y no solo la del profesor; para objetivizar el proceso y percibir elementos poco visibles para el docente en diversas etapas (Medadian \& Nezhadansari, 2015). En este proyecto de traducción, el experto en el área 
e iniciador del encargo puede proporcionar valiosa información sobre el desarrollo de la competencia temática del estudiante y su evolución durante el proyecto y los compañeros del grupo de trabajo pueden aportar información sobre los componentes actitudinales involucrados y valorarlos formalmente.

Queda pendiente comprobar empíricamente estas ideas en la próxima implementación del proyecto y en la aplicación de los diversos instrumentos de evaluación diseñados.

\section{REFERENCIAS}

Ahumada, P. (2001). La evaluación en una concepción de aprendizaje significativo. Valparaíso: Ediciones Universitarias Universidad de Valparaíso. Barkley, E., Cross, K. P. \& Howell Major, C. (2007). Técnicas de aprendizaje colaborativo. Madrid: Ministerio de Educación y Ciencia/Morata.

Boud, D. \& Falchikov, N. (2006). Aligning assessment with long-term learning. Assessment and Evaluation in Higher Education, 31(4), 399-413. Recuperado de https://goo.gl/WaMKKQ

Bruffee, K. A. (1995). Sharing our toys: Cooperative learning versus collaborative learning. Change, 27(1), 12-18. https://doi.org/10.1080/00091383 .1995 .9937722

Halliday, M. A. K. \& Hasan, R. (1985). Language, context, and text: aspects of language in a social-semiotic perspective. Oxford: Oxford University Press.

House, J. (1997). Translation Quality Assessment: Past and Present. New York: Routledge.

Hurtado, A. (2007). Traducción y Traductología. Introducción a la Traductología. Madrid: Cátedra.

Kelly, D. (2002). La Competencia Traductora: Bases para el Diseño Curricular. Puentes 1, 9-19. Recuperado de https://goo.gl/ZhQkbh

Kiraly, D. (2000). A Social Constructivist Approach to Translator Education. Empowerment from Theory to Practice. Manchester, UK: St. Jerome Publishing.

Kussmaul, P. (1995). Training the Translator. Philadelphia: Benjamins.

Larose, R. (1989). Théories Contemporaines de la traduction. Québec, Canadá: Presses de la Université de Québec.

Lazo, R. M. \& Zachary, M. (2008). La enseñanza de la traducción centrada en el estudiante. Onomázein, 1, 17, 173-181. Recuperado de https:// goo.gl/XJjtpw

Marais, K. (2013). Constructive Alignment in Translator Education: Reconsidering Assessment for Both Industry and Academy. Translation and Interpreting, 5(1), 13-24. Recuperado de https://goo.gl/2AMCc1

Marco, J. (2004). ¿Tareas o Proyectos? Senderos que se bifurcan en la adquisición de la competencia traductora. Trans, 8, 75-88. Recuperado de https://goo.gl/6BHEG5

Matthews, R. (1996). Collaborative learning: Creating knowledge with students. En R. J. Menges, M. Weimer \& Associates (Eds.), Teachíng on solid ground: Using scholarship to improve practice (pp. 101-124). San Francisco: Jossey-Bass.

Medadian, G. \& Nezhadansari, D. (2015). A Summative Translation Quality Assessment Model for Undergraduate Student Translations: Objectivity Versus Manageability. Studies about Languages, 26, 40-54. doi: http://dx.doi.org/10.5755/j01.sal.0.26.12421

Mossop, B. (2001). Revising and Editing for Translators. Manchester: St. Jerome.

Muñoz, A. (2015). El desarrollo de la competencia traductora a través de la socialización con el experto en la materia: una experiencia didáctica. The Journal of Specialised Translation, 23, 333 - 351. Recuperado de https://goo.gl/YL2AVr

Navarro, I., Pertegal Felices, M. L., Gil, D., González, C. \& Jimeno-Morenilla, A. (2011). El aprendizaje basado en proyectos como estrategia didáctica y pedagógica para estimular el desarrollo de competencias profesionales. En M. T. Tortosa Ybáñez, J. D. Álvarez Teruel \& N. Pellín Buades (Eds.), IX Jornades de xarxes d'investigació en docència universitària: Disseny de bones pràctiques docents en el context actual (p. 895). Alicante: Universidad de Alicante.

Nord, C. (1991). Text Analysis in Translation. Theory, Method and Didactic Application of a Model for Translation Oriented Text Analysis [Translated from the German by Christiane Nord and Penelope Sparrow]. Amsterdam/Atlanta GA: Rodopi. 
Nord, C. (2009). El funcionalismo en la enseñanza de traducción. Mutatis Mutandis: Revista Latinoamericana de Traducción, 2(2), $209-243$.

Parra, S. (2005). La revisión de traducciones en la Traductología: Aproximación a la práctica de la revisión en el ámbito profesional mediante el estudio de casos y propuestas de investigación (Tesis doctoral). Universidad de Granada, Granada, España.

Rodríguez, B. (2013). El enfoque constructivista en la didáctica de la revisión de traducciones. Revista de Estudios Filológicos, 25. Recuperado de https://goo.gl/pMBnt6

Rojas, 0. (2004). El portafolio y la evaluación del proceso en traducción. Letras, 36, 27-64. Recuperado de https://goo.gl/qaLYhJ

Schleiermacher, F. (2000). Sobre los diferentes métodos de traducir. Madrid: Gredos.

Toledo, M. (2010) El resumen automático y la evaluación de traducciones en el contexto de la traducción especializada. Frankfurt am Main: Peter Lang.

Umaña, O. \& Suárez, M. (2011). Descripción y explicación del diseño de instrumentos que miden la competencia traductora y terminología en traductores profesionales. EAN, 70, 20-41 Recuperado de https://goo.gl/n9hAUE

Venuti, L. (1995). The Translator's Invisibility. A History of Translation. London and New York: Routledge.

Waddington, C. (1999). Estudio comparativo de diferentes métodos de evaluación de traducción general (Inglés-Español). Madrid: Publicaciones de la Universidad Pontificia Comillas. 


\section{APÉNDICE A}

\section{GUIÓN DE PROYECTO}

\section{¿En qué consiste el proyecto?}

Este proyecto fue adjudicado en la línea “Estrategias de aprendizaje activo”, en la convocatoria 2015 para Proyectos de Innovación Docente (PID) de la Universidad de Santiago de Chile, con una duración de dos años. El objetivo general es diseñar, ejecutar y evaluar un proyecto colaborativo para la formación de traductores. A grandes líneas, algunos expertos que trabajan en distintas unidades académicas de la Universidad asignarán a los estudiantes encargos de traducción reales y los asesorarán a través de interacciones presenciales y virtuales, para aportar al desarrollo de la competencia temática (Kelly, 2002). El proyecto será implementado en la asignatura de Traducción Especializada en Ciencias Sociales Inglés-Español ( $4^{\circ}$ año) de la carrera de Licenciatura en Lingüística Aplicada a la Traducción de esta Universidad.

¿Cuáles son sus bases teóricas?

Postulados de Kiraly (2000), quien plantea que desde la perspectiva del socioconstructivismo, la manera más efectiva de lograr que un estudiante adquiera competencias profesionales es entregarle oportunidades para que interactúe con sus compañeros y con expertos y así construya su aprendizaje de manera colectiva e individual.

Enfoque funcionalista de Nord (2009), el cual sitúa la actividad traductora en las interacciones comunicativas que se producen en situaciones delimitadas con respecto a espacio y tiempo.

Aprendizaje colaborativo: "Se produce cuando los alumnos y los profesores trabajan juntos para crear el saber... es una pedagogía que parte de la base de que las personas crean significados juntas y que el proceso las enriquece y las hace crecer" (Matthews, 1996). De acuerdo con Bruffee (1995), la meta del aprendizaje colaborativo es desarrollar a personas reflexivas, autónomas y elocuentes

\section{¿Cómo se seleccionan los textos?}

Los textos seleccionados son del área de Ciencias Sociales, específicamente de Estudios Internacionales y Educación, facilitados por expertos que se desempeñan en esas áreas en nuestra universidad. Son textos que los especialistas requieren usar frecuentemente y que tienen la limitación de estar en inglés, con una extensión de aproximadamente 3000 palabras. Se seleccionaron los textos teniendo en cuenta los siguientes criterios (Umaña \& Suárez, 2011):

- Nivel de especialidad alto.

- Diversidad en los problemas de traducción y de terminología.

- Extensión apropiada para evidenciar subcompetencias traductoras y terminológicas.

- Densidad terminológica.

La profesora encargada del curso realizó un análisis previo de cada texto, para identificar los segmentos, términos o palabras en los cuales los traductores participantes podrían tener problemas de traducción. Este instrumento se denomina texto etiquetado (Umaña \& Suárez, 2011). 
¿Cuál será la forma de trabajo?

El proyecto tendrá una duración de un mes. Primero se formarán los grupos de trabajo. Luego, cada grupo recibirá un texto al azar con su correspondiente encargo de traducción. Cada grupo comenzará con la revisión de su texto y realizará la documentación necesaria en fuentes especializadas escritas, tanto impresas como virtuales. Luego iniciarán la traducción. Con la mediación de la profesora, los participantes entrarán en contacto con los especialistas. Durante el proceso de trabajo, los participantes llevarán un registro de sus actividades e interacciones con el experto (monitoreo) a través de un medio virtual (por definir). Además de reunirse con él/ ella personalmente en las ocasiones que definan de común acuerdo, también podrán establecer contacto por correo electrónico y/o un medio virtual. El mínimo de sesiones presenciales con el experto es dos. Es importante mencionar que la frecuencia y días de contacto los establecerán directamente los estudiantes con el experto en la primera reunión de trabajo.

Durante el periodo del proyecto, los estudiantes no recibirán otras asignaciones del curso y las clases constituirán una instancia de reunión para coordinación y discusión de los grupos y una sesión de consultas y resolución de problemas con la profesora.

Nota: durante las clases, la profesora también observará el desempeño de los grupos y la interacción entre sus integrantes, para ayudar a la buena dinámica de trabajo

¿Cómo se formarán los grupos?

Para la realización del trabajo colaborativo se conformarán grupos formales, que se constituyen para lograr un objetivo más complejo que puede requerir varias sesiones o semanas de trabajo (Barkley, Cross \& Howell Major, 2007). Se organizarán de acuerdo con la selección libre de los estudiantes, considerando un máximo de tres integrantes. Todos los participantes del grupo deben comprometerse activamente a trabajar juntos para alcanzar los objetivos señalados. Si un miembro del grupo realiza una tarea asignada al grupo, mientras los otros se dedican a mirar, no se realiza un aprendizaje colaborativo. Si todos los miembros del grupo reciben la tarea o si todos realizan actividades diferentes que, juntas, constituyen un único proyecto mayor, todos los estudiantes deben contribuir más o menos por igual (Barkley et al., 2007).

De acuerdo con Navarro, Pertegal Felices, Gil, González y Jimeno-Morenilla (2011), para asegurar un intercambio enriquecedor entre los diferentes alumnos, una participación igualitaria y un trabajo coordinado, es necesario diseñar una serie de roles con unas funciones encargadas a cada uno de ellos. Los roles y las funciones se pactarán junto con los alumnos, con la intención de que se impliquen más en la responsabilidad que tienen con su equipo

Se asignarán los siguientes roles entre ellos:

1. Encargado de proyecto, quien liderará el proyecto de traducción y velará por el cumplimiento del encargo, además de organizar el trabajo del grupo.

2. Encargado de terminología, quien será responsable de llevar el registro de los términos consultados tanto en fuentes escritas como aquellos revisados con el experto y de preocuparse de la uniformidad terminológica del texto.

3. Encargado de comunicaciones, quien establecerá y mantendrá el contacto con el experto y llevará el registro de las interacciones y otras informaciones pertinentes para el desarrollo del proyecto. 
Cabe mencionar que todos los integrantes del grupo deberán traducir y revisar el texto de trabajo, independiente del rol que tengan dentro del grupo.

¿Cómo se evaluará el trabajo del proyecto?

El proyecto de traducción tendrá las siguientes evaluaciones:

- Informe de efectividad según encargo basado en rúbrica, complementado por observaciones del experto. (Evaluación formativa procesual y sumativa final)

- Evaluación técnica del profesor basada en rúbrica, orientada a la actuación de la competencia temática. (Evaluación formativa procesual y sumativa final)

- Coevaluación basada en escala de apreciación complementada con comentarios, orientada a la actuación de la competencia temática. (Evaluación sumativa final)

- Se entregará información más específica sobre los instrumentos de evaluación una vez finalizada la etapa de diseño).

¿Cuándo termina el proyecto?

El proyecto se deberá completar dentro del plazo de un mes. La profesora indicará la fecha correspondiente a la entrega del encargo por escrito y con un respaldo electrónico. El resultado será un portafolio de textos traducidos, certificados por el profesor y por el asesor experto en cuanto a efectividad según el encargo, que se pondrá a disposición de cada iniciador del encargo para los fines que estime pertinentes.

\section{APÉNDICE B \\ EJEMPLO PRUEBA DE DIAGNÓSTICO}

\section{THE CONTEXT OF LEARNING IN ACADEMIC DEPARTMENTS (PAUL RAMSDEN)}

(Chapter 13). Extraído de Ramsden, P. \& Entwistle, N. (1981). Effects of Academic Departments on Students' Approaches to Studying. British Journal of Educational Psychology, 51(3), 368-383. doi: 10.1111/j.20448279.1981.tb02493.x

\section{Encargo de traducción}

La traducción de este artículo de investigación es muy importante para el trabajo que se desarrolla en el área de evaluación de la Vicerrectoría Académica de la Universidad de Santiago. En este trabajo se sientan las bases de una orientación hacia el aprendizaje en educación superior y se destaca la importancia de reconocer la experiencia de los estudiantes como un componente de la calidad de la enseñanza universitaria. Se utiliza como material de referencia en los cursos de formación docente de los profesores de la Universidad.

\section{Introduction}

The special concern of this chapter is with how students' perceptions of teaching, assessment, and course content and structure within the natural setting of academic departments may influence how students learn. The focus is thus not on the framework of courses and assessment itself, but rather on what the students 
construct out of this framework. How do students experience the effects of the context of learning at university? The following three extracts from research interviews serve to illustrate the pervasive influences of teaching and assessment on students' attitudes towards studying and on their approaches to learning.

\section{Diagnostic Test}

1. Define the following concepts in your own words (in English or Spanish)

1. Construction of learning

2. Surface approach (to learning)

3. Deep approach (to learning)

4. Approaching to study inventory

5. Course perception questionnaire

2. Answer the following questions (in English or Spanish)

1. How students' perceptions may influence their approaches to study?

2. Explain one reason why students may display a poor understanding of assessments

3. Which is the most important quality of a lecturer that influences students' attitudes towards learning?

4. What strategy can be beneficial to students in all subject areas in order to improve their learning process?

\section{APÉNDICE C}

\section{REGISTRO DE FORTALEZAS Y DEBILIDADES (PROFESOR)}

Grupo:

Texto:

\begin{tabular}{|l|l|l|}
\hline $\begin{array}{l}\text { Dominio: } \\
\text { Comprensión del texto original }\end{array}$ & Contenido & Fidelidad \\
\hline Fortalezas & & Dominio del tema \\
\hline Debilidades & & \\
\hline
\end{tabular}




\begin{tabular}{|c|c|c|c|c|}
\hline $\begin{array}{l}\text { Dominio: } \\
\text { Producción del texto meta }\end{array}$ & Título & Cohesión & Sintaxis & Precisión léxica \\
\hline Fortalezas & & & & \\
\hline Debilidades & & & & \\
\hline
\end{tabular}

\begin{tabular}{|l|l|l|}
\hline Dominio: & Registro & Propósito \\
\hline Producción del texto meta & & Ortografía \\
\hline Fortalezas & & \\
\hline Debilidades & & \\
\hline
\end{tabular}

\begin{tabular}{|c|c|c|c|}
\hline $\begin{array}{c}\text { Dominio: } \\
\text { Transferencia }\end{array}$ & $\begin{array}{c}\text { Eficiencia en la } \\
\text { resolución del problemas }\end{array}$ & $\begin{array}{c}\text { Estrategias para } \\
\text { evitar interferencias }\end{array}$ \\
\hline Fortalezas & & \\
\hline Debilidades & & \\
\hline
\end{tabular}




\section{APÉNDICE D}

\section{REGISTRO DE FORTALEZAS Y DEBILIDADES (EXPERTO)}

\section{Experto:}

\section{Grupo:}

\section{Texto:}

\begin{tabular}{|l|c|c|}
\hline Dominio del tema & Claridad en la exposición \\
de las ideas & $\begin{array}{c}\text { Utilización de fuentes } \\
\text { de documentación sugeridas }\end{array}$ \\
\hline Fortalezas & \\
\hline Debilidades & & \\
\hline
\end{tabular}

\begin{tabular}{|c|c|c|c|}
\hline $\begin{array}{c}\text { Adaptación al } \\
\text { destinatario }\end{array}$ & $\begin{array}{l}\text { El texto funciona como } \\
\text { un texto especializado } \\
\text { en el área respectiva. }\end{array}$ & $\begin{array}{l}\text { El vocabulario utilizado } \\
\text { se asemeja al de textos } \\
\text { de esta área escritos } \\
\text { en español. }\end{array}$ & $\begin{array}{l}\text { La estructura del texto } \\
\text { se asemeja a la que se utiliza } \\
\text { generalmente en textos de } \\
\text { esta área escritos en español }\end{array}$ \\
\hline \multicolumn{4}{|l|}{ Fortalezas } \\
\hline \multicolumn{4}{|l|}{ Debilidades } \\
\hline $\begin{array}{l}\text { Interacción } \\
\text { con asesor }\end{array}$ & $\begin{array}{l}\text { Interés del } \\
\text { estudiante }\end{array}$ & $\begin{array}{l}\text { Asertividad y } \\
\text { adecuación de } \\
\text { las preguntas }\end{array}$ & $\begin{array}{l}\text { Comportamiento profesional } \\
\text { (puntualidad, trato } \\
\text { respetuoso, cumplimiento } \\
\text { de compromisos) }\end{array}$ \\
\hline \multicolumn{4}{|l|}{ Fortalezas } \\
\hline Debilidades & & & \\
\hline
\end{tabular}




\section{APÉNDICE E}

\section{BITÁCORA REFLEXIVA}

\section{Alumnos}

Texto:

Responda las siguientes preguntas en tres momentos distintos del proceso de traducción: principio (primera semana de trabajo), mitad (final de segunda semana), término (final del proceso). Internalice las preguntas y reflexione antes de registrar sus respuestas. Elabore sus respuestas en 300 a 400 palabras en cada momento; ejemplifique. Entregue a la profesora al final del proceso.

Bitácora 1: inicio del proceso

1. ¿Cuáles son los principales problemas que anticipo en el proceso de traducción en relación con el dominio del tema?

2. ¿Cómo planifico resolverlos?

3. ¿Qué recursos teóricos, metodológicos y tecnológicos proyecto utilizar para resolver los problemas?

Bitácora 2: mitad del proceso

4. ¿Qué problemas enfrento en el proceso de traducción en relación con el dominio del tema?

5. ¿Qué recursos teóricos, metodológicos y tecnológicos utilizo para resolver estos problemas?

6. ¿Qué pienso respecto al desempeño de mi competencia temática en este proyecto?

\section{Bitácora 3: final del proceso}

7. ¿Qué pienso respecto a la calidad del texto traducido en función del dominio del tema?

8. ¿Qué habilidades deben tener los traductores de textos especializados como éste?

9. ¿Qué aprendí para el futuro respecto a la competencia temática y su desarrollo? 


\section{APÉNDICE F}

\section{EJEMPLO DE RÚBRICA DE EVALUACIÓN PROFESOR}

\section{Alumnos:}

Texto:

\section{DOMINIO 1: COMPRENSIÓN DEL TEXTO ORIGINAL}

\begin{tabular}{|c|c|c|c|c|c|}
\hline Valoración & Muy alta & Alta & Media & Suficiente & Insuficiente \\
\hline & 5 & 4 & 2 & 1 & 0 \\
\hline \multirow[t]{2}{*}{$\begin{array}{l}1 . \\
\text { Contenido }\end{array}$} & $\begin{array}{l}\text { El texto } \\
\text { traducido } \\
\text { conserva el } \\
\text { contenido del } \\
\text { texto original }\end{array}$ & $\begin{array}{l}\text { El texto } \\
\text { transmite casi } \\
\text { todas las ideas; } \\
\text { sin embargo } \\
\text { se observa } \\
\text { algún problema } \\
\text { de omisión o } \\
\text { adición de ideas }\end{array}$ & $\begin{array}{l}\text { Se observan } \\
\text { algunas } \\
\text { adiciones u } \\
\text { omisiones } \\
\text { de ideas } \\
\text { ocasionales } \\
\text { o poco } \\
\text { importantes. }\end{array}$ & $\begin{array}{l}\text { Se observan } \\
\text { adiciones y } \\
\text { omisiones de } \\
\text { ideas frecuentes } \\
\text { o importantes } \\
\text { que afectan el } \\
\text { contenido del } \\
\text { texto }\end{array}$ & $\begin{array}{l}\text { El texto presenta } \\
\text { adiciones u } \\
\text { omisiones de } \\
\text { ideas que afectan } \\
\text { seriamente el } \\
\text { contenido del } \\
\text { texto }\end{array}$ \\
\hline & 5 & 4 & 2 & 1 & 0 \\
\hline \multirow[t]{2}{*}{$\begin{array}{l}2 . \\
\text { Fidelidad }\end{array}$} & $\begin{array}{l}\text { El texto } \\
\text { transmite } \\
\text { fielmente } \\
\text { todas las ideas } \\
\text { contenidas en } \\
\text { el texto original } \\
\text { y las reproduce } \\
\text { con todos sus } \\
\text { matices. }\end{array}$ & $\begin{array}{l}\text { Se observan } \\
\text { ciertos } \\
\text { problemas } \\
\text { menores de } \\
\text { fidelidad a las } \\
\text { ideas del texto } \\
\text { original }\end{array}$ & $\begin{array}{l}\text { Se evidencian en } \\
\text { el texto algunos } \\
\text { problemas } \\
\text { de sentido } \\
\text { ocasionales }\end{array}$ & $\begin{array}{l}\text { Se observa falta } \\
\text { de precisión } \\
\text { en el texto; } \\
\text { ambigüedades } \\
\text { y matices no } \\
\text { reproducidos }\end{array}$ & $\begin{array}{l}\text { El texto } \\
\text { presenta varios } \\
\text { contrasentidos, } \\
\text { distintos } \\
\text { sentidos (matiz } \\
\text { no reproducido, } \\
\text { exageración, } \\
\text { reducción, } \\
\text { ambigüedad, } \\
\text { poca precisión, } \\
\text { connotaciones } \\
\text { negativas) }\end{array}$ \\
\hline & 5 & 4 & 2 & 1 & 0 \\
\hline $\begin{array}{l}3 . \\
\text { Dominio del } \\
\text { tema }\end{array}$ & $\begin{array}{l}\text { La claridad en } \\
\text { la exposición de } \\
\text { todas las ideas } \\
\text { evidencia un } \\
\text { sólido dominio } \\
\text { del tema. }\end{array}$ & $\begin{array}{l}\text { Se exponen con } \\
\text { claridad casi } \\
\text { todas las ideas; } \\
\text { se observa, sin } \\
\text { embargo, cierta } \\
\text { confusión sobre } \\
\text { el tema en otras }\end{array}$ & $\begin{array}{l}\text { Algunas ideas } \\
\text { se exponen con } \\
\text { claridad; sin } \\
\text { embargo otras } \\
\text { evidencian falta } \\
\text { de dominio } \\
\text { temático }\end{array}$ & $\begin{array}{l}\text { Varias ideas } \\
\text { expuestas } \\
\text { muestran } \\
\text { contradicciones } \\
\text { o falta de lógica, } \\
\text { lo que evidencia } \\
\text { problemas de } \\
\text { comprensión o } \\
\text { dominio del tema }\end{array}$ & $\begin{array}{l}\text { La excesiva falta } \\
\text { de claridad en } \\
\text { la exposición de } \\
\text { ideas es indicio } \\
\text { de problemas } \\
\text { serios de } \\
\text { dominio temático }\end{array}$ \\
\hline
\end{tabular}




\section{APÉNDICE G}

\section{EJEMPLO DE EVALUACIÓN SUMATIVA -EXPERTO TEMÁTICO}

\section{Experto:}

Grupo:

Texto:

1. DOMINIO DEL TEMA

Destacado

$-3$

\section{Bueno -}

2

\section{Suficiente}

$-1$
Insuficiente

0

El grupo demostró un dominio del tema adecuado para producir una versión traducida efectiva

Comentario:

El grupo expone con claridad los conceptos abordados en el texto original

Comentario:

El grupo se preparó, utilizó de manera efectiva las fuentes de documentación y siguió las recomendaciones hechas.

Comentario: 


\section{APÉNDICE H}

\section{PAUTA DE COEVALUACIÓN}

\section{Grupo $N^{\circ}$}

Integrante:

\begin{tabular}{|c|c|c|c|c|}
\hline INDICADORES & $\mathrm{s}$ & c & 0 & $\mathrm{~N}$ \\
\hline $\begin{array}{l}\text { 1. Mi compañero (a) cumplió puntualmente con las responsabilidades } \\
\text { asignadas. }\end{array}$ & & & & \\
\hline $\begin{array}{l}\text { 2. Mi compañero (a) contribuyó activamente al proceso de documentación } \\
\text { previo a la traducción }\end{array}$ & & & & \\
\hline $\begin{array}{l}\text { 3. Mi compañero (a) participó activamente en la búsqueda de equivalencias } \\
\text { léxicas durante el proceso de traducción }\end{array}$ & & & & \\
\hline $\begin{array}{l}\text { 4. Mi compañero (a) participó activamente en la integración de las } \\
\text { equivalencias léxicas en el texto durante el proceso de traducción }\end{array}$ & & & & \\
\hline $\begin{array}{l}\text { 5. Mi compañero lal contribuyó activamente en la resolución de los } \\
\text { problemas enfrentados durante la traducción }\end{array}$ & & & & \\
\hline $\begin{array}{l}\text { 6. Mi compañero lal participó activamente en las interacciones } \\
\text { con el experto y demostró eficiencia para obtener información que } \\
\text { le permitiera resolver los problemas enfrentados }\end{array}$ & & & & \\
\hline $\begin{array}{l}\text { 7. Mi compañero (a) participó activamente en el proceso de revisión } \\
\text { final del texto traducido }\end{array}$ & & & & \\
\hline $\begin{array}{l}\text { 8. Mi compañero (a) demostró rigurosidad y capacidad de autocrítica } \\
\text { durante el desarrollo del proyecto. }\end{array}$ & & & & \\
\hline $\begin{array}{l}\text { 9. Mi compañero desempeñó eficientemente el rol que asumió } \\
\text { dentro del grupo }\end{array}$ & & & & \\
\hline PUNTAJE TOTAL & & & & \\
\hline NOTA & & & & \\
\hline
\end{tabular}

$\mathbf{S}=$ siempre (2 puntos) $\mathbf{C}=$ casi siempre (1) 0 = ocasionalmente (0,5 punto) $\mathbf{N}=$ nunca (0 puntos) TOTAL: 18 puntos 
COMENTARIOS

(c) Los autores. Este artículo es publicado por la Revista Digital de Investigación en Docencia Universitaria del Área de Institutional Research and Effectiveness de la Dirección de Aseguramiento de la Calidad, Universidad Peruana de Ciencias Aplicadas. Este es un artículo de acceso abierto, distribuido bajo los términos de la LicenciaCreativeCommons Atribución-Compartirlgual 4.0 Internacional.I http://creativecommons.org/licenses/bysa/4.0/l, que permite el uso no comercial, distribución y reproducción en cualquier medio, siempre que la obra original sea debidamente citada. 